\title{
UTILIZACIÓN DE UNA ESCALA DE RIESGO PREOPERATORIA PARA GUIAR PROFILAXIS ANTIEMÉTICA EN CIRUGÍA MAYOR AMBULATORIA. ESTUDIO PROSPECTIVO, ALEATORIO. RESULTADOS PRELIMINARES
}

\author{
Nardiello Mallea Michela Andrea ${ }^{1}$, Marchant Kempt Joel², Retamales Rojas Katerin ${ }^{3}$, Orrego Arroyo Julio ${ }^{3}$, San Martín \\ Rodrigo $^{3}$, Webar Valdivieso Javier ${ }^{4}$ \\ Residente 2do año Anestesiología Universidad de Concepción. \\ Anestesiólogo Universidad de Concepción. \\ Interno 6to año Medicina Universidad de Concepción. \\ Residente 3er año Anestesiología Universidad de Concepción.
}

Introducción: Las náuseas y vómitos post operatorios (NVPO) son una complicación frecuente presentándose en $10-30 \%$ de los pacientes. Se recomienda utilizar profilaxis antiemética basada en factores de riesgo, utilizando escalas de predicción como la de Apfel. La evidencia es controversial respecto a si esta aproximación confiere beneficios versus una profilaxis estándar.

Objetivo General: Impacto de la aplicación de una escala de predicción de riesgo de NVPO preoperatoria y una recomendación de profilaxis basada en factores de riesgo en la frecuencia de NVPO.

Material y Métodos: Estudio prospectivo, aleatorio. Realizado entre junio y agosto de 2016, en pacientes ASA I y II programados a cirugía mayor ambulatoria (CMA) en el Hospital Regional Guillermo Grant Benavente.Los pacientes fueron evaluados y categorizados según score de Apfel de 1-4. El grupo control recibió cuidados habituales a consideración del anestesiólogo. En el grupo intervención, el anestesiólogo recibió una recomendación por escrito incluyendo el score de Apfel del paciente y un esquema profilaxis antiemética según su riesgo calculado. Todos los pacientes fueron evaluados en la UCPA por un evaluador ciego. Los datos fueron ingresados y analizados en SPSS. El estudio cuenta con la aprobación del comité de ética de la facultad de medicina de la universidad de concepción. Estudio prospectivo, aleatorio. Realizado entre junio y agosto de 2016, en pacientes ASA I y II programados a cirugía mayor ambulatoria (CMA) en el Hospital Regional Guillermo Grant Benavente. Los pacientes fueron evaluados la mañana de la cirugía y categorizados según score de Apfel de 1-4. El grupo control recibió cuidados habituales a consideración del anestesiólogo. En el grupo intervención, el anestesiólogo recibió un sobre con una recomendación por escrito incluyendo el score de Apfel del paciente y una recomendación de profilaxis antiemética según su riesgo calculado. Todos los pacientes fueron evaluados en la UCPA por un evaluador ciego. Los datos fueron ingresados y analizados en SPSS. El estudio cuenta con la aprobación del comité de ética de la facultad de medicina de la Universidad de Concepción.

Resultados: Se randomizaron 31 pacientes, 16 a grupo control y 14 a grupo intervención, se perdieron 2 pacientes por no cumplir criterios para CMA. Del total de pacientes, 22 fueron de sexo femenino, la edad promedio fue de 39 años (rango 18-60), peso promedio 71,85 (rango 50-103 $\mathrm{kg}$ ). Los grupos fueron comparables en sexo, edad, IMC, y factores de riesgo de NVPO. Los resultados de NVPO precoces para el grupo control e intervención fue de 8 y 6 respectivamente. NVPO totales en $24 \mathrm{~h}$ fue de 12 en el grupo control y 7 en el grupo intervención, ambos no estadísticamente significativos (p 0,431884). Los resultados se resumen en la tabla 1. La intensidad de las náuseas a los 30 minutos fue menor en el grupo intervención versus el grupo control $(\mathrm{p}<$ $0,05)$. Sin diferencias a los 60 minutos y a las 24 horas.

Conclusiones: No se encontraron diferencias entre los grupos en náuseas, vómitos ni NVPO precoces, tardíos y totales. Tampoco en necesidad de medicamentos antieméticos de rescate, estadía abreviada en UCPA y hospitalización no programada. Esto puede tener relación con el alto uso de medicamentos antieméticos en los cuidados estándar por los anestesiólogos de nuestro hospital, sumado a la falta de potencia del estudio actual para alcanzar diferencias significativas. Existen diferencias en la intensidad de las náuseas a los 30 minutos, lo que pudiese ser relevante sobre todo para pacientes de alto riesgo. 